\title{
A Comprehensive Model for Performance Analysis of APD-based FSO Systems using M-PPM Signaling in Atmospheric Turbulence
}

\author{
Hien T. T. Pham, Ngoc T. Dang \\ Department of Wireless Communications, \\ Posts and Telecommunications Institute of Technology, Hanoi, Vietnam \\ Correspondence: Ngoc T. Dang, ngocdt@ptit.edu.vn \\ Manuscript communication: received 04 September 2012, accepted 20 April 2013
}

\begin{abstract}
In this paper, a model of Gaussian pulse propagation over atmospheric turbulence channel is used to comprehensively analyze the performance of APD-based free-space optical communication (FSO) systems using M-PPM signaling. This model is able to simultaneously take into account all effects of atmospheric turbulence including attenuation, intensity fluctuation, and pulse broadening, which has not been considered in previous works. In addition, the impacts of APD shot noise, background noise, and thermal noise are included in our analysis. The numerical results show that the main factor that limits the system performance is intensity fluctuation. However, when $M$ is large enough, optical pulse is so short that the effect of pulse broadening becomes dominant. By using APD receiver, bit-error rate is improved significantly. Finally, based on channel capacity, we are able to determine the maximum transmission length of the system.
\end{abstract}

Keywords- Free-space optical communications (FSO), pulse-position modulation (PPM), avalanche photodiode (APD), atmospheric turbulence.

\section{INTRODUCTION}

Over past few years, free-space optical communication (FSO) has attracted considerable attention for a variety of applications thanks to its cost-effectiveness, licensefree, quick deployment and flexibility [1]. There are recently some efforts to introduce FSO to the first-mile access environment, especially in remote/isolated areas with low density user or in those areas where cable installation is difficult or delayed [2].

A major impairment over FSO links is the atmospheric turbulence, a phenomenon occurs as a result of the variations in the refractive index due to inhomogeneities in temperature and pressure changes [3]. These index inhomogeneities can deteriorate the quality of the received signal and can cause fluctuations in both the intensity and the phase of the received signal. These fluctuations can lead to an increase in the link error probability, which limits the performance of FSO systems [4]. Moreover, propagating pulses may be influenced by pulse broadening owing to turbulence. Two possible causes that exist for this pulse broadening are scattering (dispersion) and pulse wander (fluctuations in arrival time) [5].

Since lasers are normally intensity modulated and detection is noncoherent (i.e., direct detection), previous FSO studies have often employed $M$-ary pulse position modulation (PPM) as an energy-efficient transmission method [6, 7]. In addition, PPM avoids adaptive threshold adjustment required in on-off keying (OOK) [7]. Moreover, avalanche photodiode (APD) is also proposed to be used at the receiver to further improve the system performance [6]. According to our survey, the performance of PPM-based FSO systems has not been analyzed comprehensively. First, previous works only consider the impact of intensity fluctuation while pulse broadening effect caused by turbulence has not been taken into account. In addition, the optical pulse representing for a PPM symbol is often assumed to be a square pulse. This assumption is not realistic especially for short pulse that is generally used in PPM systems. Moreover, in many works, system performance is evaluated versus energy per information bit [8] or signal-to-noise ratio (SNR) [9], without considering the negative impact of receiver noise.

In this paper, we, therefore, propose to use a realistic model of Gaussian pulse propagation in order to comprehensively analyze the impact of atmospheric turbulence on the performance of APD-based FSO systems using PPM. This model should be able to analyze all the effects of atmospheric turbulence, including attenuation, intensity fluctuation, and pulse broadening. It is worth noting that, as PPM symbol detection is noncoherent, phase fluctuation due to turbulence does not affect the system performance. Additionally, numerous noises including APD shot noise, background noise, and thermal noise will be included in the analysis.

The rest of the paper is organized as follows. Section 2 presents the model of atmospheric turbulence channel. The model of PPM-based FSO system and its performance analysis are presented in Section 3 and Section 4, respectively. Section 5 shows the numerical results and discussion. Finally, Section 6 concludes the paper. 


\section{Atmospheric Turbulence Channel}

\subsection{Log-nornal Channel Model}

The atmosphere is not an ideal communication channel. Inhomogeneities in the temperature and pressure of the atmosphere lead to refractive index variations along the transmission path, which is commonly known as atmospheric turbulence. It produces a variety of phenomena such as frequency selective attenuation, absorption, scattering, and scintillation. When an optical beam propagates through the atmosphere, the signal intensity as observed with an optical detector at the end of the path is fluctuated randomly. This is referred to as scintillation, and it is also the major impairment of FSO communication systems.

It is difficult to determine the probability density function (pdf) for the intensity fluctuations under arbitrary atmospheric conditions and beam parameters. However, based on scintillation statistics, various mathematical models have been proposed such as lognormal [10], Gamma [11] or Gamma-Gamma [12] distribution. In this paper, as we consider weak turbulence scenario, the log-normal distribution model is adopted.

A random variable $B$ has a log-normal distribution if the random variable $A=\ln B$ has a normal (i.e., Gaussian) distribution. Thus, if the amplitude of the random path gain $B$ is $I$, the optical intensity $I=B^{2}$ is also lognormally distributed in this case. Consequently, the fading channel coefficient, which models the channel from the transmit aperture to the receive aperture, is given by

$$
h=\frac{I}{I_{m}}=\exp (2 X),
$$

where, $I_{m}$ is the signal light intensity, actually at the transmitter, without turbulence; $I$ is the signal light intensity, actually at the receiver, with turbulence. Logamplitude $X$, which is the identically distributed normal random variable with mean $\mu_{x}$ and standard deviation $\sigma_{x}$, can be expressed as

$$
f_{x}(X)=\frac{1}{\sqrt{2 \pi} \sigma_{x}} \exp \left(-\frac{\left(X-\mu_{x}\right)^{2}}{2 \sigma_{x}^{2}}\right) .
$$

To ensure that the fading does not attenuate or amplify the average power, we normalize the fading coefficients so that $\mathrm{E}(h)=1$. Doing so requires the choice of $\mu_{x}=-\sigma_{x}^{2}$. Substituting Equation (1) in Equation (2), the distribution of light intensity fading induced by turbulence, which is also a log-normal distribution, can be expressed as

$$
f_{I}(h)=\frac{1}{\sqrt{8 \pi} h \sigma_{x}} \exp \left(-\frac{\left[\ln (h)+2 \sigma_{x}^{2}\right]^{2}}{8 \sigma_{x}^{2}}\right),
$$

where $\sigma_{x}^{2}$, under weak turbulence conditions, are given by [13]

$$
\sigma_{x}^{2}=0.124\left(\frac{2 \pi}{\lambda}\right)^{7 / 6} L^{11 / 6} C_{n}^{2}
$$

where $\lambda$ is the wavelength and $L$ is the link distance in meters. $C_{n}^{2}$ stands for the refractive index structure coefficient.

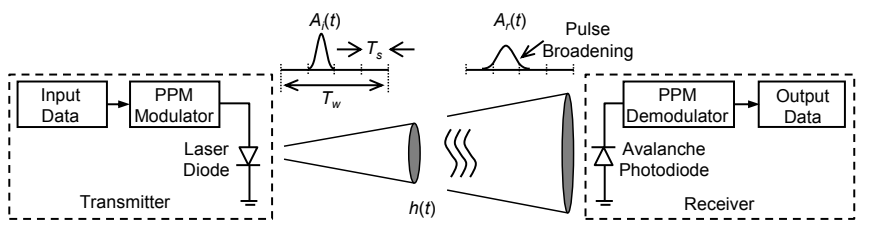

Figure 1. A point-to-point APD-based FSO system using PPM signaling.

\subsection{Pulse Propagation Model}

To obtain explicit expressions concerning the timedomain spreading of a pulse wave propagating through atmospheric turbulence, let us assume that the input waveform is the Gaussian pulse. The amplitude of the Gaussian pulse is described by

$$
A_{i}(t)=\sqrt{P_{p}} \exp \left(-\frac{t^{2}}{T_{0}^{2}}\right),
$$

where $P_{p}$ and $T_{0}$ are the peak power and the half-width (at the $1 / e$ point) of the input pulse, respectively.

As we assumed above, the fading does not attenuate or amplify the average power, the optical pulse at the receiver located at distance $L$ from the transmitter hence can be expressed as

$$
A_{r}(t)=\sqrt{P_{p} \frac{A}{\pi \theta^{2} L^{2}} \exp (-\beta L)} \frac{T_{0}}{T_{b}} \exp \left(-\frac{t^{2}}{T_{b}^{2}}\right),
$$

where $T_{b}=\sqrt{T_{0}^{2}+8 \alpha}$ is the received pulse half-width. $A$ is receiver aperture area. $\theta$ and $\beta$ are beam divergence angle in mrad and attenuation coefficient in $\mathrm{km}^{-1}$, respectively. The parameter $\alpha$ is given by [5]

$$
\alpha=\frac{0.3908 C_{n}^{2} L L_{0}^{5 / 3}}{c^{2}}
$$

where $L_{0}$ is the outer scale of turbulence and $c$ is the light velocity.

\section{APD-BASED FSO SYSTEM USING PPM SIGNALING}

A point-to-point APD-based FSO communication system using PPM is shown in Figure 1. In the transmitter side, input data is first modulated by a PPM modulator. Each block of $b=\log _{2} M$ data bits is mapped to one of $M$ possible symbols $\left(s_{0}, s_{1}, \ldots, s_{M}\right)$, where $M=2^{b}$. The symbol intervals, $T_{w}$, are divided into $M$ time-disjoint time slots and an optical pulse with constant power of $P_{u}$ is sent in one of these $M$ time slots while remaining $M-1$ time slots are empty. For the bit rate of $R_{b}$ bit per second, the symbol intervals have a duration given by $T_{w}=b / R_{b}$, and time slots have the duration of $T_{s}=T_{w} / M$.

Assuming that optical pulse sent in one time slot is a Gaussian pulse with mathematical model shown in Equation (5), the relation between the peak power of Gaussian pulse $\left(P_{p}\right)$ and the average power $\left(P_{u}\right)$ is given 
by

$$
P_{p}=\frac{\sqrt{2} T_{s}}{\sqrt{\pi} T_{0}} P_{u}
$$

The derivation of Equation (8) is presented in detail in the Appendix.

At the receiver, a receiving aperture followed by a len collects and focuses the incident beam onto an APD. The APD converts the collected optical power to an electrical current. This current is proportional to the intensity of the received signal at the receiver scaled by the detector responsivity, $\Re(\mathrm{A} / \mathrm{W})$. Finally, at the PPM demodulator, integrated photocurrents over $M$ slots are compared, and the position of the slot with the highest current determines the transmitted symbol and recovers the binary data.

\section{Performance Analysis}

\subsection{Signal and Noise}

At the receiver, the photocurrent $\left(I_{u}\right)$ corresponding to the symbol $s_{u}(0 \leq u \leq M-1)$ can be expressed as

$$
I_{u}=\Re \bar{g} h P_{u}+z_{u},
$$

where $\Re$ and $\bar{g}$ are the responsivity and average gain of APD, respectively. $h$ is the fading channel coefficient. $P_{u}$ is the average transmitted power at time slot $u$, without intensity fluctuation. As we assume that $\mathrm{E}(h)=1, P_{u}$ can be considered as the average received power at time slot $u$, without intensity fluctuation. The receiver noise $\left(z_{u}\right)$, including shot noise and thermal noise, is additive Gaussian noise with zero mean and variance as follows

$$
\sigma_{I_{u}}^{2}=2 e \Re F \bar{g}^{2}\left(h P_{u}+P_{b}\right) \Delta f+\frac{4 k_{B} T}{R_{L}} \Delta f,
$$

where $e$ is the electron charge; $k_{B}$ is Boltzmann constant; $T$ is the absolute temperature; $R_{L}$ is the load resistor; $P_{b}$ denotes optical background power; and $\Delta f=B_{e} / 2$ is the effective noise bandwidth. $F$ is the excess noise factor of the APD, which is given by

$$
F=\zeta \bar{g}+\left(2-\frac{1}{\bar{g}}\right)(1-\zeta),
$$

where $\zeta$ denotes the ionization factor.

\subsection{Bit Error Rate}

In this subsection, we present the method to calculate the bit error rate (BER) of the FSO system using $M$-PPM and APD. Denoting $P_{e}$ as the symbol error probability, the bit error rate of the system then can be derived as

$$
B E R=\frac{M}{2(M-1)} P_{e} .
$$

We assume that the transmitted data is large enough that the probabilities of sending any symbols are the same. Without the loss of generality, we also assume that symbol $s_{0}$ is transmitted. By using union bound technique, the upper bound to the instantaneous symbol error probability can be expressed as

$$
\begin{aligned}
P_{e} & \leq 1-\operatorname{Pr}\left\{I_{0}>I_{u} \mid u \in\{1, \ldots, M-1\}, s=s_{0}\right\} \\
& \leq \sum_{u=1}^{M-1} \operatorname{Pr}\left\{I_{u} \geq I_{0} \mid s=s_{0}\right\}=(M-1) \operatorname{Pr}\left\{I_{1} \geq I_{0} \mid s=s_{0}\right\} \\
& \leq(M-1) \int_{0}^{\infty} f_{I}(h) Q\left\{\frac{\mu_{I_{0}}-\mu_{I_{1}}}{\sqrt{\sigma_{I_{0}}^{2}+\sigma_{I_{1}}^{2}}}\right\} d h,
\end{aligned}
$$

where $s$ represents the transmitted symbol. $Q($.$) is the$ $Q$ function. $\mu_{I_{0}}, \sigma_{I_{0}}^{2}, \mu_{I_{1}}$, and $\sigma_{I_{1}}^{2}$ are means and variances of $I_{0}$ and $I_{1}$, respectively. Their values are derived from Equation (9) and (10) as

$$
\begin{aligned}
\mu_{I_{0}} & =\Re \bar{g} h P_{0}, \\
\mu_{I_{1}} & =\Re \bar{g} h P_{1}, \\
\sigma_{I_{0}}^{2} & =2 e \Re F \bar{g}^{2}\left(h P_{0}+P_{b}\right) \Delta f+\frac{4 k_{B} T}{R_{L}} \Delta f, \\
\sigma_{I_{1}}^{2} & =2 e \Re F \bar{g}^{2}\left(h P_{1}+P_{b}\right) \Delta f+\frac{4 k_{B} T}{R_{L}} \Delta f .
\end{aligned}
$$

When symbol $s_{0}$ is transmitted, there is a Gaussian pulse appearing at the receiver during time slot 0 . Assuming that slot 0 starts at the time $t=0$, the average received power at time slot 0 and time slot 1 hence can be expressed as

$$
P_{0}=\frac{1}{T_{s}} \int_{0}^{T_{S}}\left|A_{r}(t)\right|^{2} d t \text { and } P_{1}=\frac{1}{T_{S}} \int_{T_{s}}^{2 T_{s}}\left|A_{r}(t)\right|^{2} d t .
$$

where $T_{S}$ is the duration of one PPM time slot.

The integration in Equation (13) can be efficiently computed by Gauss-Hermite quadrature formula [14]; hence, BER can be computed as Equation (15). Here, $k$ is the order of approximation. $\left\{\omega_{i}\right\}$ and $\left\{x_{i}\right\}$ $(i=-k,-k+1, \ldots,-1,1,2, \ldots, k)$ are the weight factors and the zeros of the Hermite polynomial, respectively.

\subsection{Channel Capacity}

Channel capacity $(C)$ is the tightest upper bound on the amount of information that can be reliably transmitted over a communications channel. Characterizing the capacity of the optical channel provides a useful bound on the data rates achievable with any modulation and coding scheme, thus serving as a benchmark for assessing the performance of a particular design. The capacity will be a function of the received optical signal and noise powers, the modulation, and the detection method.

The capacity can be divided into two categories, hard and soft decisions, depending on the type of information provided to the decoder by the receiver. In this paper, we assume the case of hard-decision, in which the receiver makes estimates of each PPM symbol passing these estimates (or hard decisions) on to the decoder. Therefore, the capacity may be expressed as a function of the probability of symbol error $\left(P_{e}\right)$. The hard-decision M-PPM channel can be modeled as an 


$$
\mathrm{BER} \approx \frac{M}{2 \sqrt{\pi}} \sum_{i=-k, i \neq 0}^{k} \omega_{i} Q\left(\frac{\Re \bar{g}\left(P_{0}-P_{1}\right) e^{2 \sqrt{2} \sigma_{x} x_{i}+2 \mu_{x}}}{\sqrt{2 e F \bar{g}^{2} \Re\left[\left(P_{0}+P_{1}\right) e^{2 \sqrt{2} \sigma_{x} x_{i}+2 \mu_{x}}+2 P_{b}\right] \Delta f+8 \frac{k_{b} T}{R_{L}} \Delta f}}\right)
$$

Table I

System Parameters and Constants.

\begin{tabular}{lll}
\hline \hline Name & Symbol & Value \\
\hline Boltzmann's constant & $k_{B}$ & $1.38 \times 10^{-23} \mathrm{~W} / \mathrm{K} / \mathrm{Hz}$ \\
Electron charge & $e$ & $1.6 \times 10^{-19} \mathrm{C}$ \\
Load resistor & $R_{L}$ & $50 \Omega$ \\
Receiver temperature & $T$ & $300 \mathrm{~K}$ \\
PD responsivity & $\Re$ & $1 \mathrm{~A} / \mathrm{W}$ \\
Ionization factor & $\zeta$ & 0.028 \\
Background power & $P_{b}$ & $-40 \mathrm{dBm}$ \\
Outer scale of turbulence & $L_{0}$ & $20 \mathrm{~m}$ \\
Attenuation coefficient (clear air) & $\beta$ & $0.1 \mathrm{~km}$ \\
Beam divergence angle & $\theta$ & $1 \mathrm{rad}$ \\
Receiver aperture diameter & $d_{R}$ & $8 \mathrm{~cm}$ \\
Wavelength & $\lambda$ & $1550 \mathrm{~nm}$ \\
Refractive index structure coeff. & $C_{n}^{2}$ & $3 \times 10^{-14} \mathrm{~m}^{-2 / 3}$ \\
Bit rate & $R_{b}$ & $10 \mathrm{Gbps}$ \\
\hline & &
\end{tabular}

$M$-ary input, $M$-ary output, symmetric channel; hence, capacity is given by [15]

$$
C=\log _{2} M+\left(1-P_{e}\right) \log _{2}\left(1-P_{e}\right)+P_{e} \log _{2}\left(\frac{P_{e}}{M-1}\right),
$$

where $P_{e}$ is the probability of incorrect symbol detection, which is derived from Equation (13).

\section{Numerical Results}

In this section, we investigate the performance of PPMbased FSO systems with APD receiver in terms of BER and channel capacity. The system parameters and constants used in the analysis are shown in Table I. For a fair comparison with other systems, the analysis is considered under a constraint on the average power per bit denoted as $P_{s}$. The relation between the average power per time slot $\left(P_{u}\right)$ and $P_{S}$ is given by $P_{u}=M\left(\log _{2} M\right) P_{s}$.

First, Figure 2 shows BER of APD-based FSO systems using $M$-PPM versus the received power per bit for two cases, with and without the impact of pulse broadening. It is seen that the impact of pulse broadening is considerable only when M-PPM with high level of modulation (i.e., large value of $M$ ) is used. More specifically, the power penalties (at BER of $10^{-6}$ ) due to pulse broadening are $0 \mathrm{~dB}, 0.3 \mathrm{~dB}$, and $0.8 \mathrm{~dB}$ for the FSO systems using 4-PPM, 64-PPM, and 128-PPM, respectively.

It is well known that BER is reduced when the level of modulation increases. However, as shown in Figure 3, when the effect of pulse broadening is taken into account, the increase of $M$ does not help to improve the system performance. In this example, the performance of 256-PPM/FSO system and 128-PPM/FSO system are nearly the same. This is because M-PPM/FSO systems with high value of $M$ require to use short pulse, which

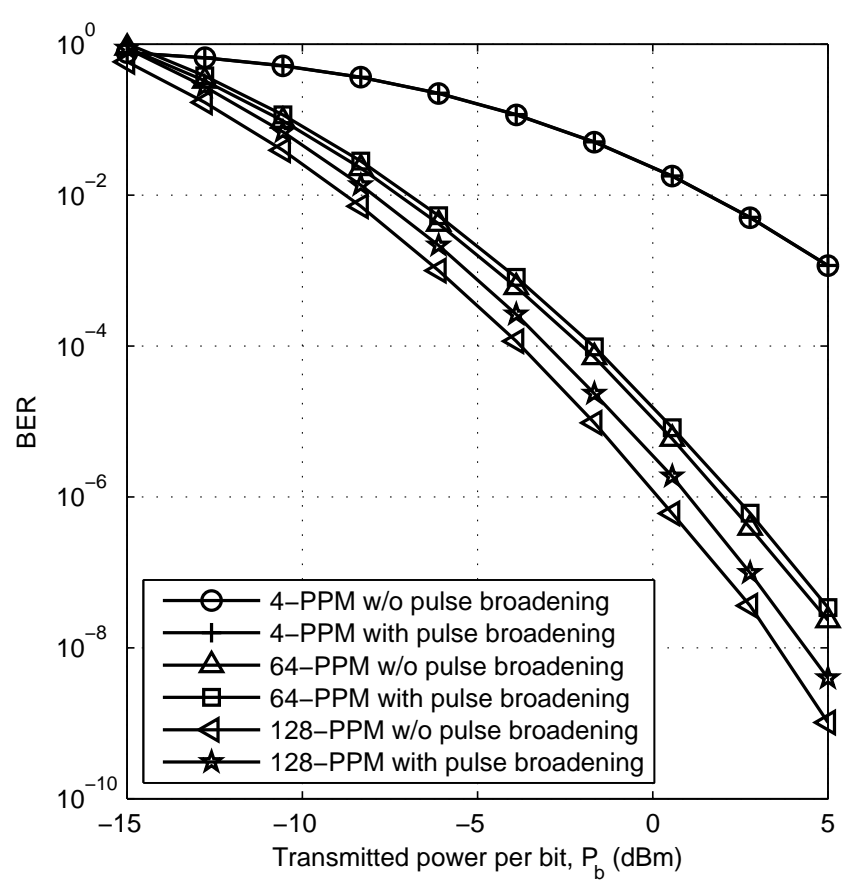

Figure 2. BER versus the transmitted power per bit with $L=2 \mathrm{~km}$ and $\bar{g}=10$.

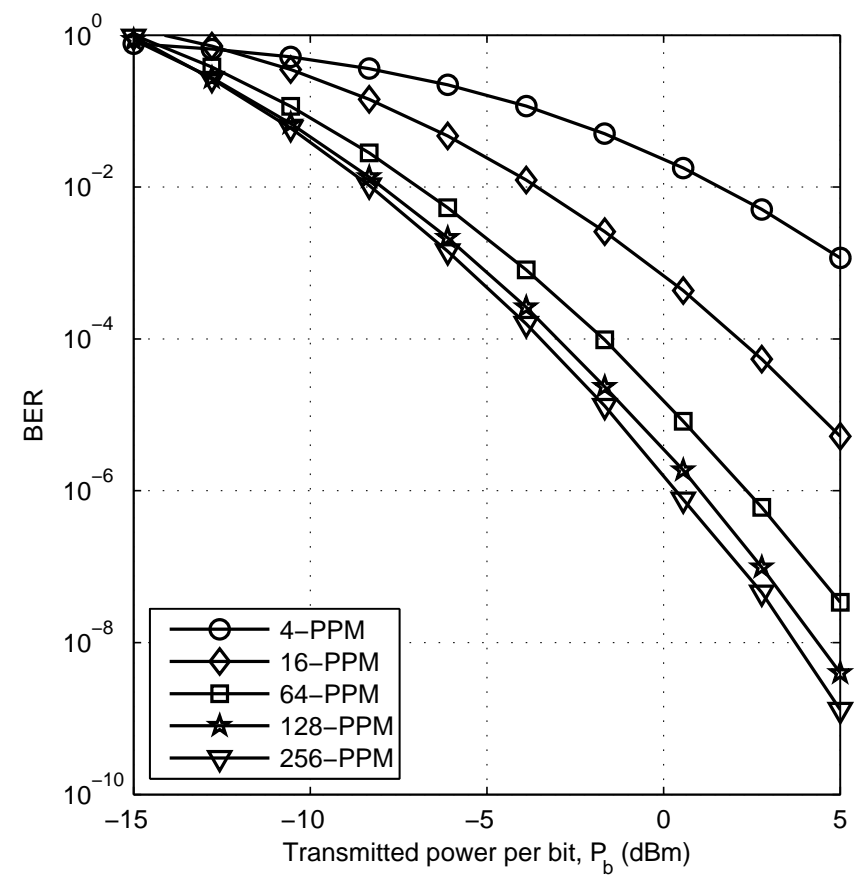

Figure 3. BER versus the transmitted power per bit with $L=2 \mathrm{~km}$ and $\bar{g}=10$. All turbulence effects are taken into account.

is more affected by pulse broadening due to atmospheric turbulence. 


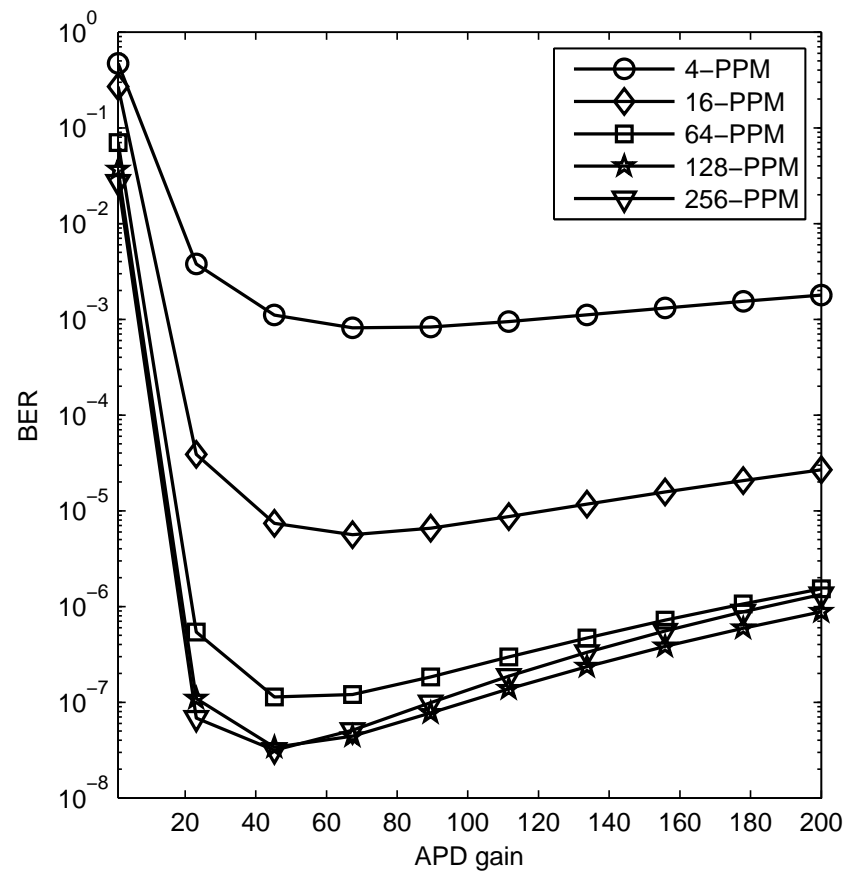

Figure 4. BER versus the average APD gain $(\bar{g})$ with $P_{s}=0 \mathrm{dBm}$. All turbulence effects are taken into account.

Figure 4 shows BER versus the average APD gain with $P_{S}=0 \mathrm{dBm}$. It is seen that BER of APD-based FSO systems using PPM are significantly reduced in comparison with the ones without APD receiver (i.e., $\bar{g}=1$ ). For all cases of M-PPM, the optimum average APD gain (i.e., the APD gain at which BER is smallest) is around 50. When APD gain is larger than 50, APD shot noise becomes considerable. As a result, the system performance cannot be improved.

Finally, we analyze the result concerning the channel capacity based on Equation (16). The result is shown in Figure 5, where channel capacity is investigated versus the transmission length with $P_{s}=-5 \mathrm{dBm}$. We define the maximum transmission length as the length at which the maximum channel capacity can be maintained. It is seen that the maximum transmission length increases from $11 \mathrm{~km}$ to $13 \mathrm{~km}$ when $M$ increases from 4 to 64 . However, as shown in the figure, the maximum transmission length cannot be further extended by increasing $M$ that is larger than 64 . This is due to pulse broadening, whose negative impact increases when the pulse width reduces.

\section{Conclusion}

We have presented a comprehensive study of the negative impact of atmospheric turbulence on the performance of $M$-PPM/FSO systems with APD receiver. A realistic model of Gaussian pulse propagation is used for analyzing the BER and channel capacity of the system. The numerical results show that, when $M \leq 128$, the main factor that limits the system performance is intensity fluctuation. However, when $M>128$, optical pulse is so short that the effect of pulse broadening

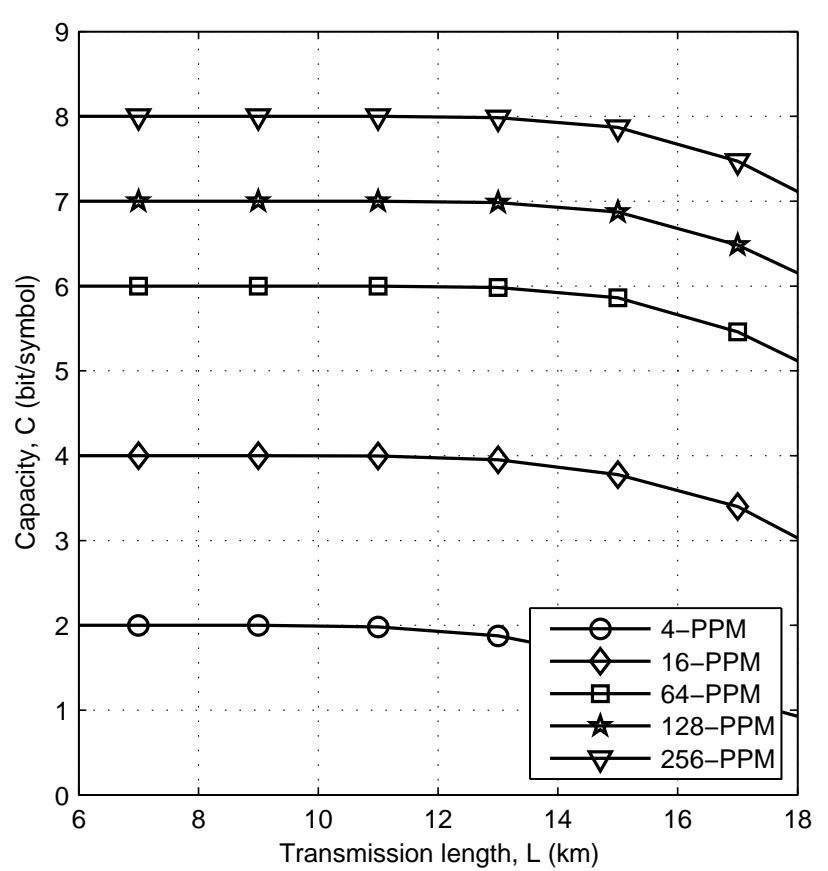

Figure 5. Capacity versus transmission length with $P_{S}=-5 \mathrm{dBm}$ and $\bar{g}=50$. All turbulence effects are taken into account.

becomes dominant. In addition, we found that APD gain of 50 is the optimum value that helps to achieve the lowest bit-error rate. Finally, based on the channel capacity, we determined the maximum transmission length of the system.

\section{APPENDIX}

\section{DeRIVATION OF Equation (8)}

The average power of transmitted Gaussian pulse, $P_{u}$, is defined as

$$
P_{u}=\frac{1}{T_{s}} \int_{-T_{s} / 2}^{T_{s} / 2}\left|A_{i}(t)\right|^{2} d t .
$$

We assume that the amplitude of transmitted Gaussian pulse is decreased so that the borders of the time slot, $-T_{S} / 2$ and $+T_{S} / 2$, can be replaced by $-\infty$ and $+\infty$ as integration limits. Equation (A.1) can be written as

$$
\begin{aligned}
P_{u} & =\frac{P_{p}}{T_{s}} \int_{-\infty}^{+\infty} \exp \left(-\frac{2 t^{2}}{T_{0}^{2}}\right) d t \\
& =P_{p} \frac{T_{0}}{\sqrt{2} T_{s}} \int_{-\infty}^{+\infty} \exp \left(-x^{2}\right) d x=P_{p} \frac{\sqrt{\pi} T_{0}}{\sqrt{2} T_{s}}(\text { A.2 })
\end{aligned}
$$

where $x=\sqrt{2} t / T_{0}$ and the Gaussian integral $\int_{-\infty}^{+\infty} \exp \left(-x^{2}\right) d x$ is equal to $\sqrt{\pi}$. From Equation (A.2), Equation (8) can be derived.

\section{REFERENCES}

[1] H. Willebrand and B. Ghuman, "Fiber optics without fiber," IEEE Spectrum, vol. 38, no. 8, pp. 40-45, 2001.

[2] Q. Liu, C. Qiao, G. Mitchell, and S. Stanton, "Optical wireless communication networks for first-and last-mile 
broadband access [invited]," Journal of optical Networking, vol. 4, no. 12, pp. 807-828, 2005.

[3] X. Zhu and J. M. Kahn, "Free-space optical communication through atmospheric turbulence channels," IEEE Transactions on Communications, vol. 50, no. 8, pp. 12931300, 2002.

[4] C. C. Davis, I. I. Smolyaninov et al., "The effect of atmospheric turbulence on bit-error-rate in an on-offkeyed optical wireless system," in Proc. SPIE, vol. 4489, 2002, pp. $126-137$

[5] C. Y. Young, L. C. Andrews, and A. Ishimaru, "Timeof-arrival fluctuations of a space-time gaussian pulse in weak optical turbulence: an analytic solution," Applied optics, vol. 37, no. 33, pp. 7655-7660, 1998.

[6] K. Kiasaleh, "Performance of apd-based, ppm free-space optical communication systems in atmospheric turbulence," IEEE Transactions on Communications, vol. 53, no. 9, pp. 1455-1461, 2005.

[7] E. J. Lee and V. W. Chan, "Part 1: Optical communication over the clear turbulent atmospheric channel using diversity," IEEE Journal on Selected Areas in Communications, vol. 22, no. 9, pp. 1896-1906, 2004.

[8] S. G. Wilson, M. Brandt-Pearce, Q. Cao, and J. H. Leveque, "Free-space optical mimo transmission with Qary PPM," IEEE Transactions on Communications,, vol. 53, no. 8, pp. 1402-1412, 2005.

[9] W. Gappmair, S. Hranilovic, and E. Leitgeb, "Performance of ppm on terrestrial fso links with turbulence and pointing errors," IEEE Communications Letters, vol. 14 , no. 5, pp. 468-470, 2010.

[10] S. M. Navidpour, M. Uysal, and M. Kavehrad, "Ber performance of free-space optical transmission with spatial diversity," IEEE Transactions on Wireless Communications, vol. 6, no. 8, pp. 2813-2819, 2007.

[11] T. A. Tsiftsis, H. G. Sandalidis, G. K. Karagiannidis, and M. Uysal, "Optical wireless links with spatial diversity over strong atmospheric turbulence channels," IEEE Transactions on Acoustics Speech and Signal ProcessingWireless Communications, vol. 8, no. 2, pp. 951-957, 2009.

[12] E. Bayaki, R. Schober, and R. K. Mallik, "Performance analysis of mimo free-space optical systems in gammagamma fading," IEEE Transactions on Communications, vol. 57, no. 11, pp. 3415-3424, 2009.

[13] I. Djordjevic, W. E. Ryan, and B. Vasic, Coding for Optical Channels. Springer, 2010.

[14] M. K. Simon and M.-S. Alouini, Digital communication over fading channels. Wiley-Interscience, 2005.

[15] H. Hemmati, Deep space optical communications. WileyInterscience, 2006

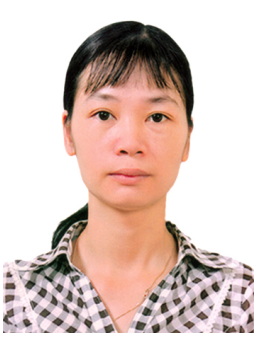

Hien T. T. Pham received the B.E degree from Hanoi University of Transport and Communications in 1999 and the M.E. degree from Posts and Telecommunications Institute of Technology (PTIT) in 2005, both in Electronics and Communications Engineering. She is currently working toward the Ph.D. degree in Telecommunication Engineering at PTIT. From 1999 to present, she has been working at PTIT as a lecturer of the Department of Wireless Communications. Her present research interests are in the area of optical and wireless communications.

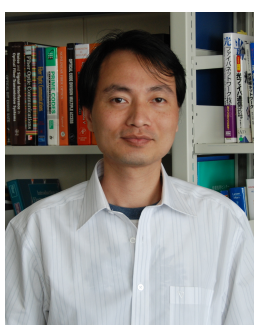

Ngoc T. Dang received a B.E. degree from Hanoi University of Technology in 1999 and an M.E. degree from Posts and Telecommunications Institute of Technology (PTIT), Vietnam, in 2005, both in electronics and telecommunications. He received a Ph.D. degree in computer science and engineering from the University of Aizu, Japan, in 2010. In 2011, he worked as an Invited Researcher at the FOTON-ENSSAT Laboratory, Universite de Rennes 1, France. In 2012, he was a JASSO Research Fellow at the Computer Communications Laboratory, University of Aizu, Japan. He is currently Deputy Head of the Department of Optical Communications at PTIT. His present research interests are in the area of optical and wireless communications. 